\title{
Nonlinear optics in silicon wire waveguides: towards integrated long-wavelength light sources
}

\author{
Bart Kuyken \\ Department of Information Technology, Ghent University
}

Supervisor(s): Roel Baets, Gunther Roelkens

Detecting small traces of pollutants in the air or analyzing blood samples has been done extensively by using light. In such a spectroscopic approach, a broadband or tunable light source reveals the absorption features of molecules which need to be detected in a sample. Strong absorption features of many molecules can be found in the so called mid-infrared region as shown in Fig 1. State-of-the-art mid-infrared spectroscopic systems use free space optics and expensive, bulky light sources. This has limited the enormous potential of mid-infrared spectroscopy to only a few sets of applications in a laboratory environment.

However by using integrated optics where on-chip lightwave circuits are used to guide and filter light, these systems could be fit on a chip measuring only a few square mm, unlocking the through potential of this wavelength region. Indeed it has been shown that silicon chips can be used to guide light, split and filter light. The use of silicon chips has many advantages not the least because such ?photonic? silicon chips are fabricated with the same equipment as used for the mass fabrication of electronic silicon chips. However, the generation of light itself in these silicon chips remains a large challenge. In this research is shown that by using nonlinear optics, mid-infrared light can be generated on such a chip. An on-chip broadband light source is demonstrated as well as a tunable wavelength source based on a silicon chip, both operating in the mid-infrared. This is a major step towards the realization of chip-scale spectroscopic systems.

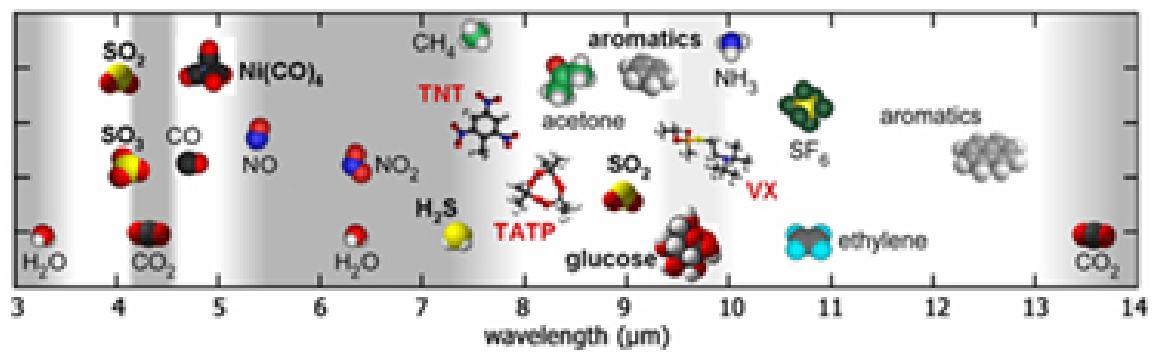

B. Kuyken acknowledges the Flemish Research Foundation, Vlaanderen for a doctoral fellowship. This work was partly carried out in the framework of the Methusalem ?Smart Photonic Chips,? FP7-ERC-INSPECTRA and FP7-ERC-MIRACLE. 